\title{
Heat and Mass Transfer in Air-Fed Pressurised Suits
}

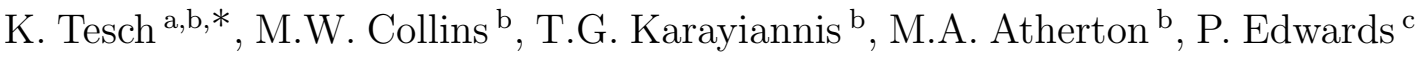 \\ ${ }^{a}$ Gdańsk University of Technology, Fluid Mechanics Department \\ ul. G. Narutowicza 11/12, 80-952 Gdańsk, Poland \\ b Brunel University, School of Engineering and Design \\ Uxbridge, Middlesex UB8 3PH, U.K. \\ c UKAEA Culham Division, Culham Science Centre \\ Abingdon, Oxfordshire OX14 3DB, U.K.
}

\begin{abstract}
Air-fed pressurised suits are used to protect workers against contamination and hazardous environments. The specific application here is the necessity for regular clean-up maintenance within the torus chamber of fusion reactors. The current design of suiting has been developed empirically. It is, therefore, very desirable to formulate a thermo-fluids model, which will be able to define optimum designs and operating parameters. Two factors indicate that the modelling should be as comprehensive as possible. Firstly, the overall thermo-fluids problem is three-dimensional and includes mass as well as heat transfer. The fluid field is complex, bounded on one side by the human body and on the other by what may be distensible, porous and multi-layer clothing.

In this paper, we report firstly the modelling necessary for the additional mass and heat transport processes. This involves the use of Fick's and Fourier's laws and conjugate heat transfer. The results of an initial validation study are presented. Temperatures at the outlet of the suits were obtained experimentally and compared with those predicted by the overall CFD model. Realistic three-dimensional geometries were used for the suit and human body. Calculations were for turbulent flow with single- and twocomponent (species) models.
\end{abstract}

Key words: Heat transfer, mass transfer, two-component turbulent flows, protective suits

\section{Introduction}

\subsection{Overall project}

Air-fed suits that form the basic application for this study are designed to protect workers against surface and airborne radioactive contamination. The suits are air-fed in the sense of having an independent 'umbilical' supply of air for ventilation. They are also pressurised to avoid inhalation of external contaminated air if the integrity of the suits is compromised. The pressurised suits do not, however, protect workers against liquids, chemicals, fire and radiation and cannot be used in non-life sustaining atmospheres.

The current research project has the following objectives: to develop a comprehensive thermo-fluids model of the entire system, comprising suit, microclimate, human surface and metabolism; to carry out validation processes to justify confidence in the model's predictive ability; and so to

\footnotetext{
* Tel. +485834728 06; Fax +48 583472020.

Email address: krzyte@pg.gda.pl (K. Tesch).
}

provide a more scientific basis for specifying optimum suit designs and operating procedures. The context, of course, is to achieve a greater assurance of the thermal comfort of pressurised suit workers. This paper reports among others a comparison between predictions and measurements of the temperature of the outlet air from a suit, an important indicator of the overall thermal balance of the system.

Fusion reactors (here the Joint European Torus [JET] at Culham, near Oxford) have periodic shutdowns for maintenance, refurbishment, decontamination of the internal torus surfaces and where needed, disassembly of facilities. Tasks that cannot be done by remote handling during shutdown periods have to be carried out by human workers. These workers enter the vessel only when the task can be justified and has specific approval. Also, apart from dedicated suit areas, operations may take place in a variety of temporary areas constructed from modular plants or tents. The maximum duration of a suited operation cannot be longer than 3.5 hours (UKAEA Code of Practice [9]).

A relevant example of use occurred during a week of the 1999 shutdown at JET, dedicated to manned vessel entry 
using the pressurised suits. There was a high ambient summer temperature for that week and the workers complained that they were suffering thermal discomfort (Campling et al. [1]). One individual withdrew prematurely from the vessel with heat stress.

\subsection{Fusion}

Fusion is the most widespread energy source (Samm et al. [10]; Unterberg et al. [11]) in the universe because it constitutes the stellar source energy. It takes place when light atomic nuclei join to create heavier nuclei and energy is (usually) released. Nuclear fission, on the other hand, occurs when heavy nuclei split into lighter components. The latter constitute waste products, whereas fusion has no waste from the process itself. It is only the internal structure that becomes radioactive. The most important reason for fusion research is the possibility of worldwide future energy generation. Fusion energy generation looks very attractive due to the abundance of the fuel and lack of emission of greenhouse gases: no $\mathrm{CO}_{2}$ is released. Even for the lightest elements such as deuterium (a form of hydrogen $H$ ) it takes significant energy to cause nuclei to fuse. This is because of the repulsive electrostatic force among the protons of the nucleus, the energy barrier that must be overcome being called the activation energy. The easiest fusion reaction to recreate in the laboratory is that of deuterium ${ }^{2} H$ and tritium ${ }^{3} H$

$$
{ }^{2} \mathrm{H}+{ }^{3} \mathrm{H} \rightarrow{ }^{4} \mathrm{He}(3.5 \mathrm{MeV})+n(14.1 \mathrm{MeV}) .
$$

This is partly because the activation energy is least for isotopes of hydrogen. In more detail the fusion of nuclei of deuterium and tritium results in the unstable nucleus ${ }^{5} \mathrm{H}$ which expels a neutron $n$ plus $14.1 \mathrm{MeV}$. It becomes a helium nucleus ${ }^{4} H$ (or alpha particle) with a recoil energy $3.5 \mathrm{MeV}$. Energy is liberated because a slight loss of mass results from the reaction. The overall energy liberation is 17.6 MeV, about 1700 times more than the activation energy $0.01 \mathrm{MeV}$.

Both deuterium and tritium (through lithium) are abundant and occur naturally. Deuterium is present in sea water $(0.0035 \%)$ and lithium in the earth's crust (0.004\%). Tritium is unstable and decays with a half-life of 12 years, so has to be produced by a fusion reaction involving lithium. The energy characteristics of fusion power are very attractive. The exploitable energy in 1000 litres of oil (Samm [10]) is equivalent to that in $75 \mathrm{mg}$ of ${ }^{2} \mathrm{H}$ (2 litres of water) and $225 \mathrm{mg}$ of ${ }^{6} \mathrm{Li}$ (1 $\mathrm{kg}$ of earth). However, the temperature $(1 \mathrm{eV}=11600 \mathrm{~K})$ needed to initiate the fusion of deuterium and tritium is about $100 \cdot 10^{6} \mathrm{~K}$, that is about seven times hotter than at the centre of the sun $\left(15 \cdot 10^{6} \mathrm{~K}\right)$. At a lower temperature of about $10^{4} K$ the gaseous mixture of deuterium and tritium becomes a plasma - that is the atoms become ionised (separated from their electrons) because the force binding them has been overcome. The plasma has to be confined, and there are various methods of achieving this. The most common and advanced is magnetic confinement. Here a magnetic field confines the plasma, that is, 'suspends' it within a containment vessel. The most common design of vessel is the so-called 'tokamak' a toroidal shaped magnetic chamber (figure 1,2).

The largest existing fusion reactor/torus facility is at Culham, near Oxford, UK, and is named the Joint European Torus (JET). The site management is provided by the UK Atomic Energy Authority (UKAEA). JET came into operation in 1983, and the world's first controlled release of fusion energy was achieved there in 1991.

The current stage in fusion research is described (Nuclear Fusion, 2003 [8]) as 'Breakeven', when the output power equals the input. This was demonstrated at JET in 1997. The next (future) stage is 'Ignition' in which no input power would be required, the plasma itself generating a sufficient amount of energy. A further step is that of the 'Burning Plasma', achieved when the plasma heats itself instead of being heated externally. The successor to JET is called the International Tokamak Experimental Reactor (ITER) and its construction is due to start in 2008 at Cadarache (France). It will be eight times larger than JET and is designed to enable 'Ignition' to be demonstrated. Also 'Burning Plasma' should be feasible as to reach this a reactor has to be at least two times larger than JET. ITER is designed to produce $500 \mathrm{MW}$ of power during a pulse of $400 \mathrm{sec}-$ onds, and will provide engineering and physics information for the development of a demonstration commercial torus power plant, DEMO. Its minimum estimated timescale is 30 years for initial production of electricity, with the first commercial reactor being available not before 2050 .

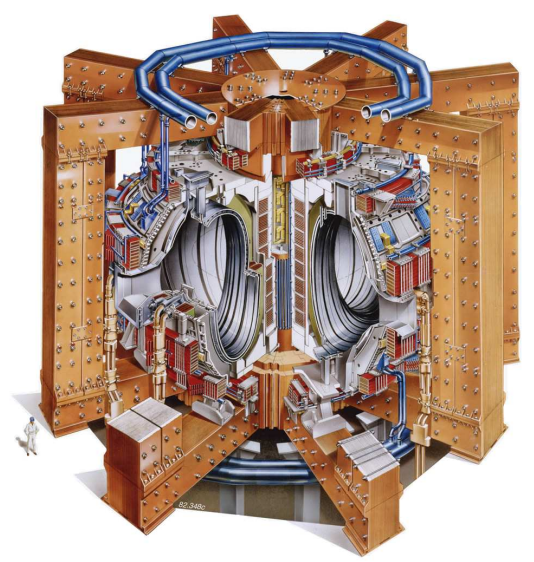

Fig. 1. Cutaway diagram of torus at JET (UKAEA - JET website)

\subsection{Tritium and other contaminants}

The plasma interacts with the component materials, of which several are in direct contact with the plasma. Certain particles may also penetrate the materials to some depth before being reflected. Irradiation caused by neutrons may cause modification of the material structure, leading to degradation of mechanical stability and thermal conductivity. 


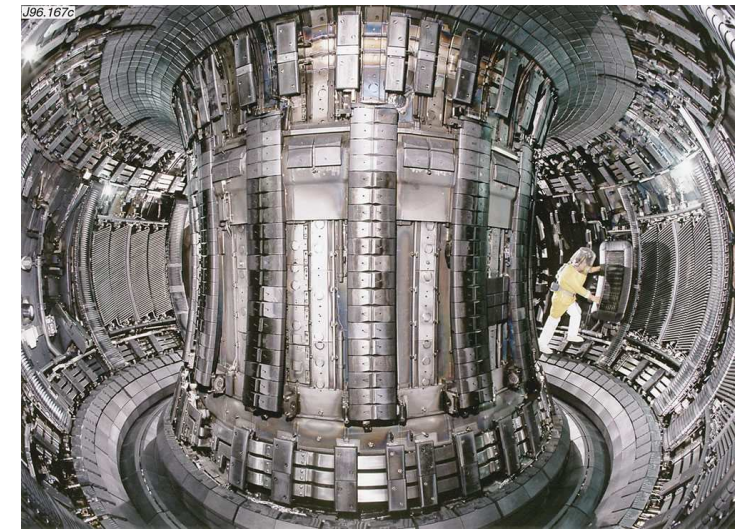

Fig. 2. Worker wearing protective suit inside torus at JET (UKAEA - JET website)

The fuel in the plasma is composed of the hydrogen isotopes deuterium and tritium. The (radioactive) tritium is stored in the wall and is responsible for radioactive storage in fusion reactors. These reactions lead to radioactive endproducts. Radioactive tritium is a weak emitter with 12.3 years half-life. The mean range in organic matter is below $6 \mu \mathrm{m}$, due to its low energy. This is small compared with the thickness of human skin at $70 \mu \mathrm{m}$ and means that as an external irradiator tritium is not highly hazardous. It may become dangerous to the human body, however, when inhaled. The effective biological half-life of tritium in the body is about 11 days. More than $95 \%$ of air-borne tritium is present in the vessel in the form of contaminated water vapour which is 10000 more radiotoxic than the tritium itself.

\subsection{Construction of pressurised suits}

Suits have to protect workers from inhalation of tritium and contamination by surface materials, especially beryllium. A suit is designed as a one-piece unit, figure 3 in section 3.1. Its fabrication material is $350 \mu m$ thick polyurethane (PU), with the suit hood being $300 \mu \mathrm{m}$ thick to improve visibility. Other approved materials such as polyvinyl chloride (PVC) can be also used. Joins in the suit fabric are radio frequency welded. Apart from its protective purpose the suit is designed to provide a supply of breathable and safe air. While there is a normal operational flow rate of $350-360 \mathrm{l} / \mathrm{min}$, a key parameter is the minimum airflow for which the suit was designed and tested.

The main airline connection is located at the rear of the internal waist-belt which is held in place within the suit by support loops. These loops are welded to the internal surface and the suit is connected to the airline by means of stainless steel Eldon couplings. The air is distributed through four ports via internal pipes to the head, arms (near wrists) and legs (near ankles). Two of the ports are placed on the trunk and two on the head. Wiring for communication is enclosed within the air-supply tubing.

The air supply system may be equipped with a chiller.
According to the UKAEA Code of Practice [9] the air supplied to the suits should be between $15-20^{\circ} \mathrm{C}$. With a chiller the temperature of the inlet air flow can be reduced from 25 to $-5^{\circ} \mathrm{C}$. However, air has to travel through an airhose between 25 and $50 \mathrm{~m}$ in length before reaching the suit and this can be problematic. Because the airhose is not well insulated thermally, the most important factor determining the temperature of the air entering the suits is the ambient temperature. For example (according to a JET experiment), with a $25 \mathrm{~m}$ airhose and $27^{\circ} \mathrm{C}$ ambient temperature it is only possible to reduce the entry air temperature to $20^{\circ} \mathrm{C}$. For a $50 \mathrm{~m}$ airhose the benefit is less, with entry air at $22^{\circ} \mathrm{C}$. In addition, it takes approximately 50 minutes before a change in chiller conditions affects the air temperature at suit entry.

Of course, the air supply itself should be free from any substances potentially affecting the health and thermal comfort of the worker. It must neither interact with ambient contaminants which could leak into the breathing zone, nor affect the breathing apparatus. Apart from the predetermined temperature, the supply air should be also dry. The pressure dew point is $5^{\circ} \mathrm{C}$ below the lowest possible temperature. If the conditions of use are not known, the pressure dew point must be at a maximum limit of $-11^{\circ} \mathrm{C}$ for a relative humidity level of $85 \%$ [9].

The hood is fitted with an emergency breather valve for use in case of failure of the air supply, meaning that this design of suit cannot be used in a non-life sustaining atmosphere. The hood also has an intercom system for communication with the control room. An additional $125 \mu \mathrm{m}$ thick polyurethane over-suit is fitted to reduce the risk of contamination to the surface of the main suit. This over-suit is removed and disposed of, before leaving the suit area. If an over-suit is being worn it must not obstruct the emergency breathing system.

A worker has his/her feet protected by wellington boots worn over the suit. If high surface contamination is expected then overshoes will be worn to protect the boots. Hands are protected by appropriate gloves, which are attached to the suit using wide adhesive PVC tape on the ends of the sleeves. Rigid tapered cuffs are fitted to ensure a tight seal. A second pair of gloves is usually worn over the first pair.

\section{Governing equations}

\subsection{Equations for the mixture}

A two-component model is likely to predict the thermofluid behaviour more accurately because of vapour present in the air of the microclimate. Vapour appears in the air, which is supplied in a dry condition, because of the respiration process and other moisture produced by the human body. The functions and values of component $i$ will be denoted by superscript $i$, where this does not indicate power. The equations for a mixture are derived from the multi- 
phase equations summation (Ishii [6]). Individual species transport equations may be written as

$$
\frac{\partial\left(\rho g^{i}\right)}{\partial t}+\nabla \cdot\left(\rho g^{i} \boldsymbol{U}\right)=\sum_{j=1}^{2} J^{i j}-\nabla \cdot \boldsymbol{j}^{i}
$$

where $g^{i}$ denotes mass fraction, the mixture density $\rho$ is defined as the sum of individual species densities $\rho^{i}$ and volume concentration, and the velocity of the mixture is represented by the sum of individual species velocities and mass fractions. The diffusive mass flux $\boldsymbol{j}^{i}=\rho g^{i} \tilde{\boldsymbol{U}}^{i}$ is the mass transfer among species and requires further modelling. The summed eq. (2) gives the mass conservation equation in the same form as that for single-component flow

$$
\frac{\partial \rho}{\partial t}+\nabla \cdot(\rho \boldsymbol{U})=0
$$

This is because the mass fluxes between phases must fulfil $J^{i j}=-J^{j i}, J^{i i}=0$. Obviously, we have $J^{i j}=0$ for a mixture.

The relationship between mass and volume fraction can be expressed as $g^{i}=\rho^{-1} \rho^{i} r^{i}$. Similarly, the momentum conservation equation for a mixture takes the following form

$$
\frac{\partial(\rho \boldsymbol{U})}{\partial t}+\nabla \cdot(\rho \boldsymbol{U} \boldsymbol{U})=\rho \boldsymbol{f}+\nabla \cdot \boldsymbol{\sigma}-\nabla \cdot \sum_{i=1}^{2} \rho g^{i} \tilde{\boldsymbol{U}}^{i} \tilde{\boldsymbol{U}}^{i}
$$

where $\rho \boldsymbol{f}=\sum_{i=1}^{2} \rho^{i} g^{i} \boldsymbol{f}^{i}$ is the density of external forces. The mixture stress tensor $\boldsymbol{\sigma}=\sum_{i=1}^{2} r^{i} \boldsymbol{\sigma}^{i}$, where the individual stress tensor is generalised to

$$
\boldsymbol{\sigma}^{i}=-p^{i} \boldsymbol{\delta}+2 \mu^{i} \boldsymbol{D}^{D i}+\mu_{v}^{i} \boldsymbol{\delta} \nabla \cdot \boldsymbol{U}^{i} .
$$

In eq. (5) $p^{i}$ is the thermodynamic pressure, $\mu^{i}$ and $\mu_{v}^{i}$ the molecular and bulk viscosity respectively, $\boldsymbol{D}^{D i}$ the strain rate tensor deviator, and $\boldsymbol{\delta}$ the Kronecker delta. Comparing eq. (4) with its equivalent for single-component flow an additional term should be noted; this is responsible for diffusion of the momentum.

The energy conservation equation for a mixture takes the form

$$
\begin{aligned}
\frac{\partial\left(\rho e_{k}\right)}{\partial t} & +\nabla \cdot\left(\rho \boldsymbol{U} e_{k}\right)=\rho \boldsymbol{f} \cdot \boldsymbol{U} \\
& +\nabla \cdot(\boldsymbol{\sigma} \cdot \boldsymbol{U}-\boldsymbol{q})-\nabla \cdot \sum_{i=1}^{2} E^{i} \boldsymbol{j}^{i}
\end{aligned}
$$

Here the total kinetic energy for the mixture $\rho e_{k}=$ $\sum_{i=1}^{2} \rho g^{i}\left(e^{i}+\frac{1}{2} U^{i 2}\right)$ and is composed of the internal energy $e^{i}$ and the macroscopic kinetic energy. The heat vector of a mixture is denoted here as $\boldsymbol{q}=\sum_{i=1}^{2} r^{i} \boldsymbol{q}^{i}$.

For a low Mach number $M a$ it is possible to simplify the equations. Because there is no need to take compressibility effects into account, the density can be assumed constant. Compressibility may be neglected in the flow of gases if $M a^{2} \ll 1$. For a considered case the maximal (local) velocity is estimated to be $5.5 \mathrm{~m} \mathrm{~s}^{-1}$, which corresponds to $M a \approx 0.016$. This value justifies the incompressibility assumption and allows the equations to be significantly simplified. If the diffusive velocity is small compared with the mixture velocity it can be assumed that all the fluid properties $\varphi^{i}$ except the concentration (mass or volume fraction) share the same fields $\forall_{i=1,2} \varphi^{i}=\varphi$. This means that the velocity, pressure and temperature fields are shared by all the components.

The mass and momentum conservation equations for the incompressible mixture take the form equivalent to those for the single-component case. To obtain this, it is assumed that all the second order terms involving diffusive velocity may be neglected. If the diffusive velocity is small then its product in eq. (4) is even smaller. The mechanical constitutive eq. (5) also simplifies to its single-component version. The additional term in eq. (6) is due to internal energy diffusion resulting from a concentration difference. A similar additional term is present in the Fourier - Kirchhoff (internal energy $e$ ) equation

$$
\frac{\partial(\rho e)}{\partial t}+\nabla \cdot(\rho \boldsymbol{U} e)=\phi_{\mu}-\nabla \cdot \boldsymbol{q}-\nabla \cdot \sum_{i=1}^{2} e^{i} \boldsymbol{j}^{i} .
$$

The dissipation function $\phi_{\mu}$ for the incompressible case takes the form $\phi_{\mu}=2 \mu \boldsymbol{D}^{2}$.

\subsection{Heat and mass transfer}

Fourier's law in the form of $\boldsymbol{q}=-\boldsymbol{\lambda} \cdot \nabla T$ is another constitutive equation for anisotropic fluids. If we consider the isotropy assumption the conductivity tensor $\boldsymbol{\lambda}$ may be expressed in terms of the isotropic part $\boldsymbol{\lambda}=\lambda \boldsymbol{\delta}$. Here the symbol $\lambda$ represents the thermal conductivity coefficient, and Fourier's law simplifies to

$$
\boldsymbol{q}=-\lambda \nabla T \text {. }
$$

The 'fluid' Fourier - Kirchhoff eq. (7) describes the temperature field in the fluid, and using $d e=c_{v} d T$ gives

$$
\begin{aligned}
c_{v}\left(\frac{\partial(\rho T)}{\partial t}+\nabla \cdot(\rho T \boldsymbol{U})\right)=\phi_{\mu} & +\nabla \cdot(\lambda \nabla T) \\
& -\nabla \cdot \sum_{i=1}^{2} c_{v}^{i} T \boldsymbol{j}^{i}
\end{aligned}
$$

where we do not assume that heat conductivity and capacity coefficients are constant. For solids where $\boldsymbol{U}=\boldsymbol{U}^{i}=\mathbf{0}$, eq. (7) simplifies to the 'solid' Fourier - Kirchhoff equation

$$
c \frac{\partial(\rho T)}{\partial t}=S_{E}+\nabla \cdot(\lambda \nabla T)
$$

where $S_{E}$ represents internal energy sources if any are present.

Generally, the closed set of thermo-fluid equations should be solved together with eq. (10). This is called conjugate heat transfer. An advantage of this approach is that it is not necessary to know the heat transfer coefficient. The disadvantage is the need to increase the total number of elements to accommodate the additional solid volume. If storage limitations do not allow such additional elements then either the temperature or heat flux must be specified at the wall. There are at least four alternatives: specified 
temperature, specified heat flux, adiabatic (zero heat flux) or specified heat transfer coefficient. The case most commonly met with in practice is the last condition given as $q_{n 1}=h_{1}\left(T_{a}-T\right)$ and $q_{n 2}=h_{2}\left(T_{b}-T\right)$, where $T_{a}$ and $T_{b}$ are an ambient and body temperatures respectively. By involving the combined heat transfer coefficients $h_{i}$ it is possible to model the thermal resistance of the wall from the solid side. The coefficient $h_{1}$ from the torus side includes free convection and conduction through the suit layers. The coefficient $h_{2}$ from the human body side includes radiation and conduction through various clothing layers. The most general case is where $h_{i}$ is not constant, so this approach is more difficult than the conjugate heat transfer model because it introduces a new unknown function. However it does save computer resources and calculation time. The bulk heat fluxes $q_{n i}$ are just expressions of the boundary condition.

The non-uniformity of the concentration causes mass transport from regions of higher - into lower concentrations. Mass transport behaves similarly to heat transfer. Fick's law expresses the mass flux stream in the same manner as Fourier's law for a heat flux, namely $\boldsymbol{j}^{i}=-\rho \boldsymbol{D}^{i j}$. $\nabla g^{i}$. The diffusivity tensor $\boldsymbol{D}^{i j}$ is usually replaced by the kinematic diffusivity coefficient $D^{i j}$, giving Fick's law as

$$
j^{i}=-\rho D^{i j} \nabla g^{i} .
$$

This means eq. (2) can be rewritten in the same form as the Fourier - Kirchhoff eq. (9). If there are no mass sources (no chemical reactions) we have

$$
\frac{\partial\left(\rho g^{i}\right)}{\partial t}+\nabla \cdot\left(\rho g^{i} \boldsymbol{U}\right)=\nabla \cdot\left(\rho D^{i j} \nabla g^{i}\right)
$$

\subsection{Turbulent two-component flow}

Because all the variables except concentration share the same fields then most of the equations previously introduced have exactly the same form as for single-component turbulent flow. The average form of mass conservation eq. (3)

$$
\nabla \cdot \overline{\boldsymbol{U}}=0 .
$$

The Navier - Stokes equations, obtained by substituting the mechanical constitutive eq. (4) into that for conservation of momentum without an additional term, becomes the Reynolds equations

$$
\frac{\partial(\rho \overline{\boldsymbol{U}})}{\partial t}+\nabla \cdot(\rho \overline{\boldsymbol{U}} \overline{\boldsymbol{U}})=\rho \overline{\boldsymbol{f}}-\nabla \bar{p}+\nabla \cdot(2 \mu \overline{\boldsymbol{D}}+\boldsymbol{R}) .
$$

We concentrate here on the approach that uses the eddy (or turbulent) viscosity $\mu_{t}$. The Reynolds stress tensor $\boldsymbol{R}$ is described by means of the Boussinesq hypothesis $\boldsymbol{R}=$ $-\frac{2}{3} \rho k \boldsymbol{\delta}+2 \mu_{t} \overline{\boldsymbol{D}}$. The Reynolds eq. (14) takes the form of the Navier - Stokes equations (Wilcox [12])

$$
\frac{\partial(\rho \overline{\boldsymbol{U}})}{\partial t}+\nabla \cdot(\rho \overline{\boldsymbol{U}} \overline{\boldsymbol{U}})=\rho \overline{\boldsymbol{f}}-\nabla p_{e}+\nabla \cdot\left(2 \mu_{e} \overline{\boldsymbol{D}}\right)
$$

where the effective viscosity $\mu_{e}:=\mu+\mu_{t}$ and effective pressure $p_{e}:=p+\frac{2}{3} \rho k$.
The two equation standard $k-\varepsilon$ turbulence model is widely used for the calculation of turbulent flows in engineering and is adopted here. These two additional equations have to be formulated. The first is for the kinetic energy $k$ of velocity fluctuations, and comes from the Reynolds stress transport equation

$$
\begin{aligned}
\frac{\partial(\rho k)}{\partial t} & +\nabla \cdot(\rho k \overline{\boldsymbol{U}})=2 \mu_{t} \overline{\boldsymbol{D}}^{2} \\
& +\nabla \cdot\left(\left(\frac{\mu_{t}}{\sigma_{k}}+\mu\right) \nabla k\right)-\rho \varepsilon .
\end{aligned}
$$

The second is for the dissipation $\varepsilon$ of the kinetic energy, and is analogous in form to that for $k$, namely

$$
\begin{aligned}
\frac{\partial(\rho \varepsilon)}{\partial t} & +\nabla \cdot(\rho \varepsilon \overline{\boldsymbol{U}})=C_{\varepsilon 1} \frac{\varepsilon}{k} 2 \mu_{t} \overline{\boldsymbol{D}}^{2} \\
& +\nabla \cdot\left(\left(\frac{\mu_{t}}{\sigma_{\varepsilon}}+\mu\right) \nabla \varepsilon\right)-C_{\varepsilon 2} \rho \frac{\varepsilon^{2}}{k} .
\end{aligned}
$$

The averaged concentration transport equation may be obtained from eq. (12) using the eddy diffusivity hypothesis

$$
\frac{\partial\left(\rho \bar{g}^{i}\right)}{\partial t}+\nabla \cdot\left(\rho \bar{g}^{i} \overline{\boldsymbol{U}}\right)=\nabla \cdot\left(\rho D_{e} \nabla \bar{g}^{i}\right)
$$

where the effective diffusivity $D_{e}$ may be represented as a function of the eddy viscosity and the turbulent Schmidt number $S c_{t}$, namely as $D_{e}:=\mu_{t} \rho^{-1} S c_{t}^{-1}+D^{i j}$.

The averaged Fourier - Kirchhoff equation for a twocomponent flow differs from that for a single-component flow, because of an additional term responsible for the diffusion of internal energy

$$
\begin{array}{r}
c_{v}\left(\frac{\partial(\rho \bar{T})}{\partial t}+\nabla \cdot(\rho \bar{T} \overline{\boldsymbol{U}})\right)=2 \mu \overline{\boldsymbol{D}}^{2} \\
+\nabla \cdot\left(\lambda_{e} \nabla \bar{T}+\rho \bar{T} \sum_{i=1}^{2} c_{v}^{i} D^{i j} \nabla \bar{g}^{i}\right)+\rho \varepsilon .
\end{array}
$$

Here the effective conductivity $\lambda_{e}$ arises from the eddy diffusivity hypothesis. It is obtained, using the turbulent Prandtl number $P r_{t}$, as $\lambda_{e}:=\mu_{t} c_{v} P r_{t}^{-1}+\lambda$. We assumed that

$$
\rho \overline{\sum_{i=1}^{2} c_{v}^{i} D^{i j} \nabla g^{i}} \approx \rho \bar{T} \sum_{i=1}^{2} c_{v}^{i} D^{i j} \nabla \bar{g}^{i}
$$

which means that the correlation of the temperature and the concentration fluctuation are neglected. This is the easiest way to compensate for the lack of a consistent model.

\subsection{Closed system of equations}

The system of ten scalar equations is composed of the mass conservation eq. (13), the Reynolds equation with Boussinesq's hypothesis (15), concentration transport eq. (18), equations of transport of $k$ (16) and $\varepsilon(17)$, Fourier - Kirchhoff eq. (19), eddy viscosity relation $\mu_{t}=C_{\mu} \rho \frac{k^{2}}{\varepsilon}$ and finally the algebraic relation between mass fractions $\bar{g}^{2}=1-\bar{g}^{1}$. The system is then closed for the ten unknown functions $\overline{\boldsymbol{U}}, \bar{p}, k, \varepsilon, \mu_{t}, \bar{T}, \bar{g}^{1}, \bar{g}^{2}$. The 'standard' set of five constants is given by $\sigma_{k}=1, \sigma_{\varepsilon}=1.3, C_{\mu}=0.09$, $C_{\varepsilon 1}=1.44, C_{\varepsilon 2}=1.92[12]$. 


\section{Results and comparison}

\subsection{Flow Domain}

Figure 3 shows a photograph of a typical worker in a pressurised suit. A realistic reconstructed geometry is given in figure 4 . The flow domain is defined by the microclimate, that is the volume which lies between the inner surface of the suit and the human surface plus undergarment. This flow domain has been discretised into 600000 elements.

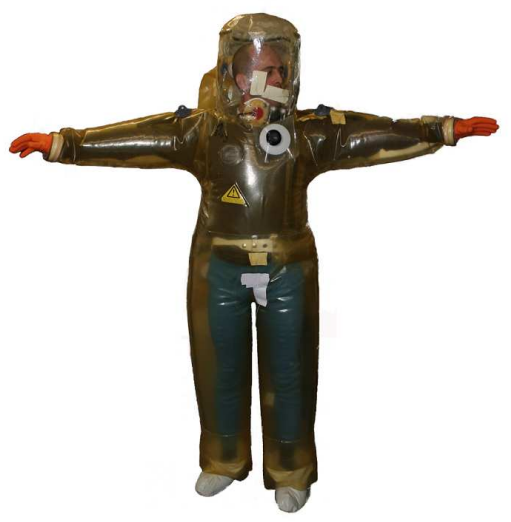

Fig. 3. Worker in protective suit

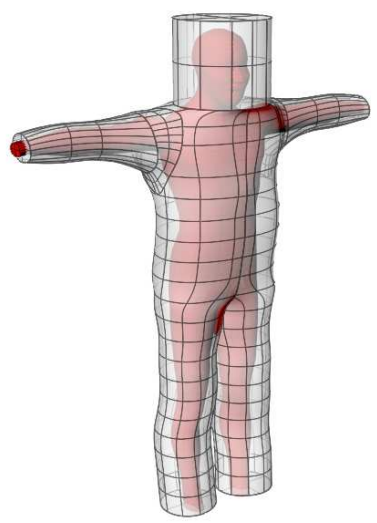

Fig. 4. Reconstruction geometry for worker/suit combination of fig. 3

\subsection{Boundary conditions}

The engineering interpretation of the boundary conditions is:

(i) Inlet - the suit presented in figure 4 has six inlets: two above the head, two near the wrists and two near the ankles. The volumetric flow rate of $390 \mathrm{l} / \mathrm{min}$ at $290.7 K$ is assumed to be uniformly divided between all six inlets. Over the usual volumetric flow range of $370-410 \mathrm{l} / \mathrm{min}$ no significant difference was found in the predicted outlet temperatures. The turbulence intensity defined as $\tau_{t}:=\frac{1}{\bar{U}} \sqrt{\frac{2}{3} k}$ was taken to be $1 \%$ and the viscosity ratio $\frac{\mu_{t}}{\mu}=1$, that is a low turbulent intensity. Equations for eddy viscosity enable the inlet $k$ to be determined in the form $k=\frac{3}{2} \bar{U}^{2} \tau_{t}$, with the dissipation rate given by the equation $\varepsilon=C_{\mu} \rho \frac{k^{2}}{\mu}$.

(ii) Outlet - there are four outlets: two above the head (where the outlet temperatures $T_{\text {out }}$ have been measured) and two at the lower part of the back. A constant static pressure is specified for each outlet.

(iii) Symmetry - because of the symmetry of the geometry only half of the computational domain has been considered. At the plane of symmetry this means that the normal component of velocity equals zero $(\hat{n} \cdot \boldsymbol{U}=0)$ and all scalar values $\varphi$ must fulfil $\hat{n} \cdot \nabla \varphi=0$, where $\hat{n}$ represents a unit vector normal to the surface.

(iv) Wall - all the velocity components equal zero. The bulk heat flux $q_{n i}$ is specified by means of the combined heat transfer coefficient $h_{i}$ and the ambient temperature.

(a) Suit - ambient temperatures $T_{a}=292 \mathrm{~K}$.

(b) Body - ambient temperature in this case equals the body temperature. There is a constant heat transfer rate between the human body and its environment due to convection, conduction and radiation. Body (core) temperature is dependent, among other factors, on metabolic rate, environment and clothing. The difference between the body core and skin temperatures allows the elimination of some of the metabolic heat so that the body reaches an 'equilibrium' temperature. A simple way of calculating this temperature and its consequent rate of change has been applied based on thermal balance considerations. This enables predictions to be made based on simulating the relevant thermal processes. Normal body temperature $T_{b 0}$ is about $310 \mathrm{~K}$ and this is achieved when there is thermal balance between the amount of heat produced by the body and losses due to a number of different causes. The heat storage $q_{S}$ may be expressed as (Henane et al. [4]; Gonzalez et al. [2]; Havenith $[3])$

$$
\begin{aligned}
q_{S}= & q_{M}-q_{W}-q_{\text {rad }}- \\
& q_{\text {conv }}-q_{\text {cond }}-q_{E}-q_{R}
\end{aligned}
$$

where $q_{M}$ represents metabolic rate, $q_{W}$ energy converted into external work, $q_{\text {rad }}$ and $q_{\text {conv }}$ heat gain on the skin by radiation and convection respectively from an environment characterised by the air-gap temperature $T$. Losses due to conduction from the core to the skin are denoted here by $q_{c o n d}$, skin evaporation by $q_{E}$ and respiratory heat loss from the lungs by $q_{R}$. Both $q_{E}$ and $q_{R}$ results in a loss of weight $m$. If $q_{M}-q_{W}>$ $q_{\text {rad }}+q_{\text {conv }}+q_{\text {cond }}+q_{E}+q_{R}$ then the body temperature $T_{b}$ increases and the human feels 
'hot'. Equations for $q_{M}, q_{W}, q_{\text {rad }}, q_{\text {conv }}, q_{\text {cond }}$, $q_{E}$ and $q_{R}$ may be found elsewhere (Henane et al. [4], [5]). The body temperature variation $T_{b}$ may then be described by means of the ordinary differential equation

$$
\begin{array}{r}
T_{b}^{\prime}(t)+\frac{S_{b} h}{c m} T_{b}(t)=\frac{S_{b}}{c m}\left(q_{M}(t)\right. \\
\left.-q_{W}(t)+h T(t)-q_{E}(t)-q_{R}(t)\right)
\end{array}
$$

where $S_{b}$ stands for body surface, $c$ body specific heat, $m$ body mass.

The mass fraction of vapour $g^{2}$ may be found from the relative humidity $\varphi$ by means of the well known equations $g^{2}=\frac{X}{1+X}$ where $X=0.622 \frac{p_{s} \varphi}{p-p_{s} \varphi}$.

\subsection{Heat transfer coefficient calibration}

It is possible to calibrate the heat transfer coefficient itself from the ambient side of the suit through considering empty suit measurements. We want to minimise the difference $\Delta T$ between values predicted by a simulation and those obtained from experiment.

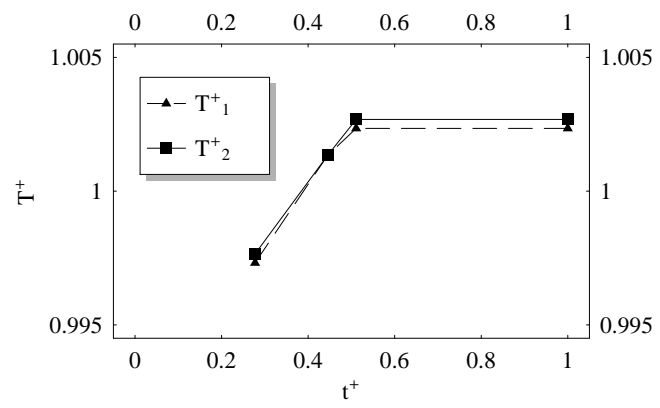

Fig. 5. Measured outlet temperatures

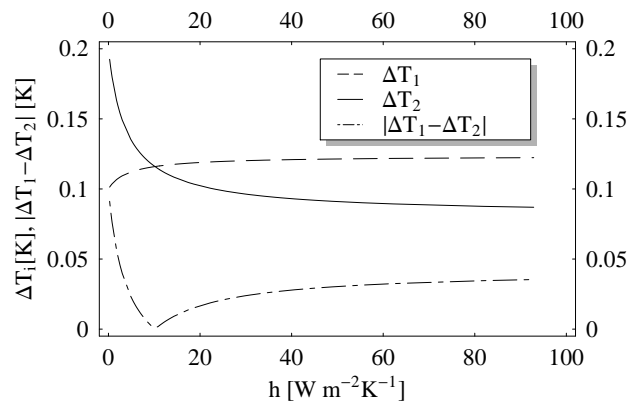

Fig. 6. Temperature differences as a function of heat transfer coefficient

Figure 5 shows measured outlet temperatures from the suit. The dimensionless temperature $T^{+}$is defined here as $T^{+}:=\frac{T}{T_{a}}$ and dimensionless time $t^{+}:=\frac{t}{\Delta t}$. The total time of measurement $\Delta t$ took about 140 min. Subscript 1 in fig. 5 and 6 represents an outlet placed at the head level and 2 at the trunk. As seen in figure 5, the temperature distributions over time are almost identical. This of course implies that for the empty suit air temperature is uniform within the whole of inner suit space. However, this is not true in the situation when a person is wearing the suit. The resulting temperature differences at the two outlets is a matter of current investigation.

In figure 6 the differences between measured and predicted temperature are plotted as a function of heat transfer coefficient. In this case it is a combined heat transfer coefficient of conduction and convection from the ambient (torus) side. It can be seen that temperature difference $\Delta T_{2}$ is more sensitive than $\Delta T_{1}$. One can deduce that further increasing the heat transfer coefficient $h$ has no significant effect on temperatures. A heat transfer coefficient value of $10 \mathrm{Wm}^{-2} \mathrm{~K}^{-1}$ was chosen. This means that this value gives equal error for both temperature differences $\left|\Delta T_{1}-\Delta T_{2}\right|=$ 0 .

\subsection{Examples of results}

Figure 7 shows the metabolic rate $q_{M}$ and production of vapour $m_{v}$ for a typical experiment performed at JET to validate CFD. The initial 36 minutes represents a stabilising period where no physical activity took place. From 36 to $49 \mathrm{~min}$. the worker had a moderate output due only to walking. This was followed by a period from 49 to $68 \mathrm{~min}$. of hard (working) activity. The final period 68 - 82 min. corresponds to cooling without any physical activity. Change of body (core) temperature from the 'equilibrium' discussed above may be observed in figure 8 for a typical human of $72 \mathrm{~kg}$ mass and $1.8 \mathrm{~m}$ height. As seen in this figure, the

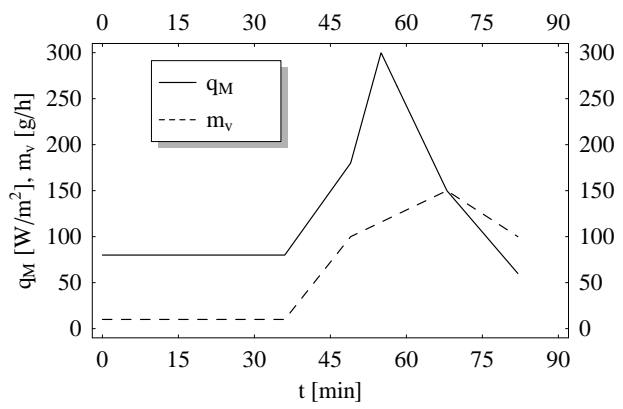

Fig. 7. Metabolic heat load and vapour production

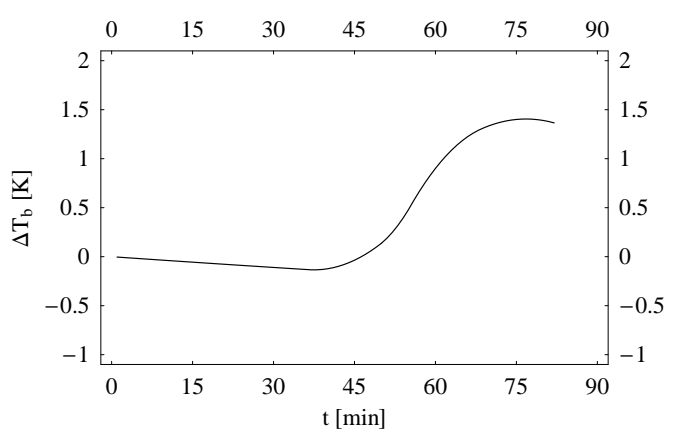

Fig. 8. Body temperature variation

core temperature is nearly constant until the person begins 
to perform work. The temperature decreases slightly when the physical activity is stopped.

Figure 9 presents a comparison between measurements of outlet temperature taken at JET and CFD calculations assuming turbulent flow. The outlet temperature was measured by means of a thermocouple placed at the outlet above the head. The dimensionless temperature is defined here as $T^{+}:=\frac{T_{\text {out }}}{T_{a}}$ where the outlet temperature from CFD results was calculated as $T_{\text {out }}:=\frac{1}{|S|} \iint_{S} T d S$. Comparative predictions for single- and two-component flow showed negligible difference in terms of outlet temperature. However it may be important to consider the two-component model for surface condensation phenomena, which are not included here. The comparison is good, and shows that the standard $k-\varepsilon$ turbulence model is adequate for the purpose of predicting the outlet temperature. Figures 10 and 11

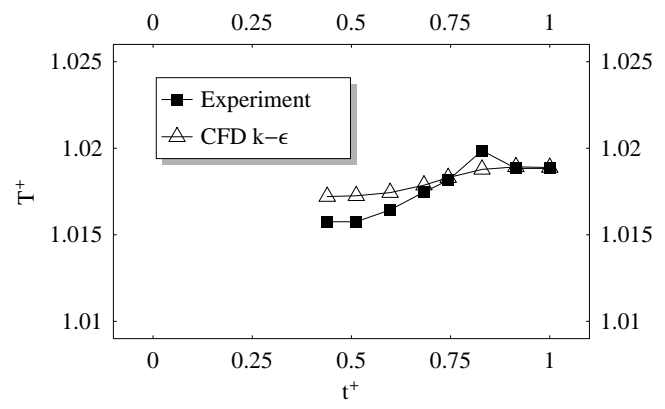

Fig. 9. Comparison of CFD predictions and measurements

show skin temperature distribution at characteristic time steps $36,42,49,56,61,68,75$ and $82 \mathrm{~min}$. Temperature variation which is caused by metabolic heat load can be observed during this time. It can also be observed that the skin temperature around the six inlets is lower than everywhere else. This is due to the low temperature of the inlet air, which successively increases with the distance from the inlet. One can also observe hot spots during hard working

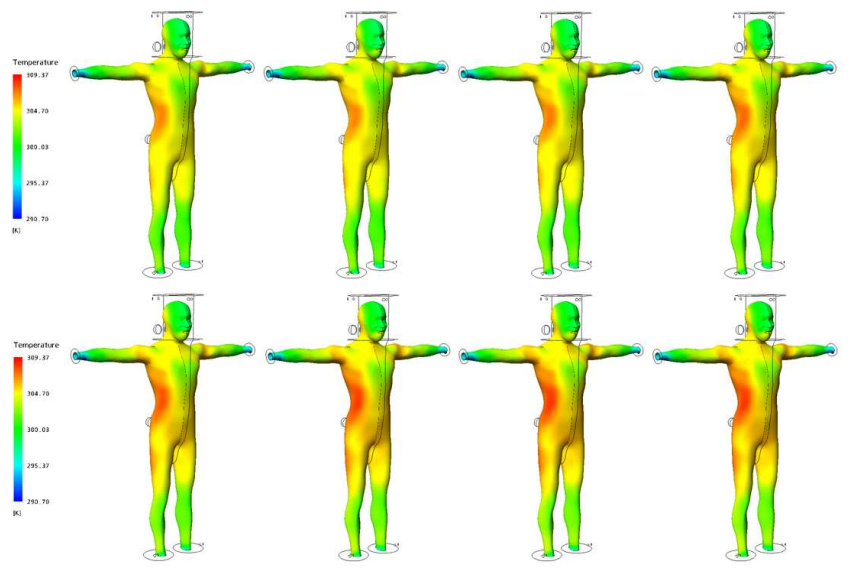

Fig. 10. CFD predictions of body temperature at time step 36 (stabilisation), 42-49 (walking), 56-61-68 (working), 75-82 (cooling) min

activity. This may be the reason of thermal discomfort of the worker.

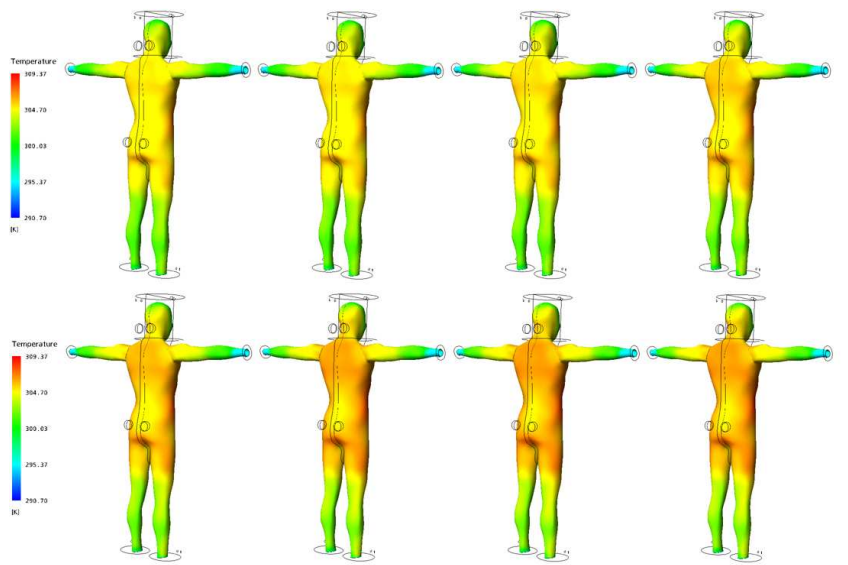

Fig. 11. CFD predictions of body temperature at time step 36 (stabilisation), 42-49 (walking), 56-61-68 (working), 75-82 (cooling) min

\section{Conclusions}

A comprehensive thermo-fluids numerical model has been developed for the microclimate in air-fed pressurised suits. It is shown that it can predict outlet temperatures in realistic three-dimensional geometries, however, further study is necessary to compare results for different turbulence models as well as the importance of two-component flows for surface condensation effects. Although, the standard $k-\varepsilon$ turbulence model has predicted satisfactory results.

While this study has concentrated on flows in the protective suits used at JET, the approach has been as generic as possible. With simple modifications the model can be applied to other types of suits.

\section{References}

[1] D.C. Campling, P.K Edwards, B. Patel, P.A. Schofield, Verification of the JET Pressurised Suit Protection Factor against Tritium, UKAEA internal report

[2] R.R. Gonzalez, T.M. McLellan, W.R. Withey, S.K. Chang, K.B. Pandolf, Heat Strain Models Applicable for Protective Clothing Systems: Comparison of Core Temperature Response, J. Appl. Physiol. 83 (1997) 1017-1032.

[3] G. Havenith, Heat Balance When Wearing Protective Clothing, Ann. Occup. Hyg. 43 (5) (1999) 289-296

[4] R. Henane, J. Bittel, Changes of Thermal Balance Induced by Passive Heating in Resting Man, J. Appl. Physiol 38 (2) (1975).

[5] R. Henane, R. Flandrois, J.P. Charbonnier, Increase in Sweating Sensitivity by Endurance Conditioning in Man, J. Appl. Physiol. 43 (1977) 822-828

[6] M. Ishii, T. Hibiki, Thermo-Fluid Dynamics of Two-Phase Flow, Springer, 2006

[7] H.W. Müller, M. Hirsch, IPP Summer University for Plasma Physics, Max-Planck-Institut für Plasmaphysik, 2005

[8] Nuclear Fusion, Parliamentary Office of Science and Technology, 192 (2003)

[9] Nuclear Industry Code of Practice for the Use of Airline Fed Pressurised Suits, UKAEA internal report (2001)

[10] U. Samm, Controlled thermonuclear fusion enters with ITER into a new era, Contemporary Physics 44 (3) (2003) 203-217 
[11] B. Unterberg, U. Samm, Overview of Tokamak Results, Fusion Science and Technology 45 (2T) (2004) 445-452

[12] D.C. Wilcox, Turbulence Modeling for CFD, DCW Industries, 1994

\section{Nomenclature}

a local speed of sound $m s$

C constant

c $\quad$ specific heat capacity $J \mathrm{~kg}^{-1} \mathrm{~K}^{-1}$

$D \quad$ strain rate tensor $s^{-1}$; diffusivity tensor $m^{2} s^{-1}$

$D \quad$ diffusivity coefficient $m^{2} s^{-1}$

e energy $J k^{-1}$

f external forces $\mathrm{kg} \mathrm{m}^{-2} \mathrm{~s}^{-2}$

$g \quad$ mass fraction

$h \quad$ heat transfer coefficient $W m^{-2} K^{-1}$

j diffusive mass flux $\mathrm{kg} \mathrm{m}^{-2} \mathrm{~s}^{-1}$

$k \quad$ kinetic energy of velocity fluctuations $m^{2} s^{-2}$

$m \quad$ mass $k g$

$M a \quad$ Mach number $M a:=\frac{U}{a}$

$\hat{n} \quad$ unit normal vector

$p \quad$ pressure; average, species and effective pressure $\mathrm{Pa}$

$\operatorname{Pr} \quad$ Prandtl number $\operatorname{Pr}:=\frac{\mu c}{\lambda}$

$\boldsymbol{q}$ heat flux vector $W m^{-2}$

$q \quad$ heat flux $W m^{-2}$

$\boldsymbol{R}$ Reynolds stress tensor $\mathrm{kg} \mathrm{m}^{-1} \mathrm{~s}^{-2}$

$r \quad$ volume fraction

$S \quad$ surface $m^{2}$; source

$S c \quad$ Schmidt number $S c:=\frac{\mu}{\rho D}$

$T$ temperature $K$

$t \quad$ time $s$

$\boldsymbol{U} \quad$ velocity vector $m s^{-1}$

$\tilde{\boldsymbol{U}} \quad$ diffusivity velocity vector $m s^{-1}$

U velocity norm $m s^{-1}$

Greek Symbols

$\boldsymbol{\delta} \quad$ Kronecker delta

$\varepsilon \quad$ dissipation of fluctuation of kinetic energy $W \mathrm{~kg}^{-1}$

$\lambda \quad$ conductivity tensor $W m^{-1} K^{-1}$

$\lambda \quad$ conductivity coefficient $W m^{-1} K^{-1}$

$\mu \quad$ viscosity $\mathrm{kg} \mathrm{m}^{-1} \mathrm{~s}^{-1}$

$\rho \quad$ density $\mathrm{kg} \mathrm{m}^{-3}$

$\boldsymbol{\sigma} \quad$ stress tensor $\mathrm{kg} \mathrm{m}^{-1} \mathrm{~s}^{-2}$

$\sigma \quad$ constant

$\tau \quad$ intensity

$\phi \quad$ dissipation function $W m^{-3}$

$\varphi \quad$ scalar; relative humidity

Superscripts

$+\quad$ dimensionless

$D$ deviatoric part

$i \quad i^{\text {th }}$ component

Subscripts

0 initial

$\mu \quad$ viscous

a ambient

$b \quad$ body

cond conductive

conv convective 